\title{
Promising effects of Chinese traditional treatment for child typhoid complicated by myocarditis
}

\author{
JING TIAN, XINJIANG AN, MINGYU FU and QINGWEN WANG \\ Department of Cardiology, Xuzhou Children's Hospital, Xuzhou, Jiangsu 221002, P.R. China
}

Received April 15, 2016; Accepted September 27, 2016

DOI: $10.3892 /$ etm.2016.3803

\begin{abstract}
The clinical effects were compared and analyzed of traditional Chinese medicine (TCM) 'Ling Gui Long Mu soup' combined with the conventional Western medications in treating child typhoid complicated by myocarditis. From July, 2010 to May, 2014, 54 children suffering from typhoid complicated by myocarditis were enrolled in the present study. The patients were divided into the observation and control groups ( $n=27$ cases per group) according to the random number table. Patients in the observation group were treated with basic Western medicine combined with TCM 'Ling Gui Long Mu soup' while patients in the control group were treated only with Western medicine. We analyzed the final curative effects in the two groups. The total effective rate in the observation group was significantly higher than that of the control group and the difference was statistically significant $(\mathrm{P}<0.05)$. The improvement rate of the syndrome in the TCM observation group was significantly higher than that in the control group and the difference was statistically significant $(\mathrm{P}<0.05)$. Although the $\mathrm{C}$-reactive protein $(\mathrm{CRP})$ and creatinine kinase-MB (CK-MB) levels in the two groups were decreased following the treatment, CRP and CK-MB levels in the observation group were further reduced, and the difference was statistically significant $(\mathrm{P}<0.05)$. In conclusion, for child typhoid complicated by myocarditis, TCM 'Ling Gui Long Mu soup' significantly improved the clinical efficiency of the treatment and improved the syndrome. Therefore, 'Ling Gui Long Mu soup' is useful in clinical practice.
\end{abstract}

\section{Introduction}

Child typhoid is an infection of the intestines that spreads to the blood and other parts of the body. It is caused by

Correspondence to: Dr Xinjiang An, Department of Cardiology, Xuzhou Children's Hospital, 18 Sudibei Road, Xuzhou, Jiangsu 221002, P.R. China

E-mail: anxinjian001@163.com

Key words: child typhoid, myocarditis, Ling Gui Long Mu soup, traditional Chinese medicine syndrome
Salmonella typhimurium. Major symptoms include acute severe diarrhea, intestinal bleeding, perforation, secondary circulation capacity reduction, systemic infection and reticular system proliferative response (1). As the typhoid bacilli enter into the intestinal tract, they start reproduction, while penetrating into the bloodstream and causing bacteremia. Salmonella typhimurium can release endotoxins that can enter myocardium (2). Phagocytic endotoxin stimulates and activates the mononuclear phagocytic system in the blood and under the mediation of such an effect, the endotoxin entered into the myocardium may create irreversible damage to the cardiac muscle cells which may eventually lead to the manifestation of myocarditis (3).

Typhoid is usually treated with a course of antibiotic medication. However, the curative effects of conventional Western medicine treatment, including antibiotics, circulation capacity maintenance and other symptomatic support treatments have not been ideal. Traditional Chinese medicine (TCM) 'Ling Gui Long Mu soup', is composed of various compounds, such as radix paeoniae alba, cassia twig, angelica, astragalus, ginseng, antelope horn, keel, oysters and liquirice (4). Evidence has shown that 'Ling Gui Long Mu soup' has promising curative effects for treating patients with heart yang deficiency (5).

Thus, the present study investigated the clinical effects of TCM 'Ling Gui Long Mu soup' combined with conventional Western medicine in treating child typhoid complicated by myocarditis.

\section{Materials and methods}

General materials. From July, 2010 to May, 201454 children suffering from typhoid complicated by myocarditis were enrolled in the present study. The inclusion criteria for the study were: In all cases the clinical symptoms and signs were consistent with the diagnostic criteria for typhoid fever, and all the cases had a positive Widal test to confirm the infection and electrocardiogram and myocardial enzymes readings supported the diagnosis of myocarditis. The exclusion criteria for the study were: Patients with primary myocarditis, cardiomyopathy, and other cardiac organic lesions were excluded. Patients with severe liver and kidney dysfunctions, the patients in serious condition with very short estimated lifetime, and patients who refused to participate in the study were also excluded.

After obtaining approval from the Xuzhou Children's Hospital Ethics Committee and informed consent from the 
family of the patients, we divided the patients into the observation and control groups, with 27 patients per group, according to random number table. The observation group comprised 16 males and 11 females, with an age range of 6-17 years (average age, $11.3 \pm 2.6$ years), 10 patients in this group suffered from dampness-heat syndrome with predominant dampness, 8 cases had dampness-heat syndrome, and 9 cases had dampness-heat syndrome with predominant heat. The control group comprised 17 males and 10 females, with an age range of 5-16 years (average age, $12.8 \pm 2.3$ years). In the control group, there were 8 cases of dampness-heat syndrome with predominant dampness, 9 cases of dampness-heat syndrome, and 10 cases of dampness-heat syndrome with predominant heat. The baseline data of the two groups were compared and differences were not statistically significant $(\mathrm{P}>0.05)$.

Method. The two groups were treated with conventional therapies, including antityphoid therapy, myocardial metabolism promotion therapy, water electrolyte balance maintenance and symptomatic support therapy. Patients in the observation group also received a daily administration of 'Ling Gui Long $\mathrm{Mu}$ soup' (Radix Paeoniae Alba, 8-16 g; cinnamon, 8-16 g; oyster, 6-12 g; keel, 6-12 g; Angelica sinensis, 6-12 g; Radix Astragali, 6-12 g; licorice, 6-12 g; American ginseng, 8-16 g; and antelope horn, 6-12 g) one pack per day for one month, taken morning and night, respectively, after water decoctum. The patients received levofloxacin capsules $(0.2 \mathrm{~g} / \mathrm{time}, \mathrm{t} . \mathrm{i} . \mathrm{d}$.) for one month. Patients in the control group were treated solely by oral administration of levofloxacin capsules $(0.2 \mathrm{~g} /$ time, t.i.d.) for one month.

Observation index. We compared and analyzed the differences in total clinical efficiency, improvement rate of TCM syndromes and recovery rate of myocardial enzymes. The criteria used for clinical effect evaluation was as follows: i) We considered that a recovery was achieved when clinical diarrhea stopped and all signs and the symptoms of infection and intoxication disappeared completely. Additionally, hemogram, liver and renal functions returned to normal, without recurrence within $72 \mathrm{~h}$; ii) we deemed our treatment as effective once clinical diarrhea considerably subsided and the symptoms and signs of infection and intoxication were considerably improved. Additionally, hemogram, liver and kidney functions significantly receded, without recurrence within $72 \mathrm{~h}$; and iii) treatment was considered to be ineffective when clinical symptoms and signs were not improved (or even aggravated and caused mortality).

The equation used to calculate 'the total efficiency' was (number of patients with recovery and effective scores/total number of cases) x $100 \%$. The improvement condition of TCM syndromes were presented as the efficacy index, calculated as: [(treatment scores before treatment - treatment scores after treatment)/treatment scores before treatment] x $100 \%$.

If the efficacy index was $\geq 95 \%$ it was considered a recovery, an efficacy index between 30 and $95 \%$ was considered effective, and for an efficacy index of $<30 \%$, the treatment was considered to be ineffective.

Statistical analysis. Statistical software package SPSS 16.0 (Chicago,IL,USA) was applied to process the data.Quantitative data were presented as means \pm standard deviation, and the t-test was used for comparisons between groups. The $\chi^{2}$ test was employed for comparisons between groups. $\mathrm{P}<0.05$ was considered to indicate a statistical significant difference.

\section{Results}

Comparison of the clinical efficiency in the groups. The number and proportion of patients with recovery and effective scores as well as the total effective rate in the observation group were significantly higher than those in the control group, and differences were statistically significant $(\mathrm{P}<0.05)$. The proportion of patients with ineffective scores in the observation group was significantly lower than the control group $(\mathrm{P}<0.05)$ (Table I).

Comparison of the improvement rate of the syndrome in the groups. The number and proportion of patients with recovery from the syndrome as well as the total effective rate of patients in the TCM observation group were significantly higher compared to those of the control group, and differences were statistically significant $(\mathrm{P}<0.05)$. Conversely, the proportion of patients with ineffective scores in the observation group was significantly smaller than the control group $(\mathrm{P}<0.05)$ (Table II).

Comparison of the changes of creatinine kinase- $M B(C K-M B)$ and $C$-reactive protein $(C R P)$ of the groups. Differences in the CRP and CK-MB levels in the two groups before the treatment were not statistically significant $(\mathrm{P}>0.05)$. However, those levels were significantly decreased in the two groups following treatment. The CRP and CK-MB levels in the observation group decreased more significantly compared to the control group, and differences were statistically significant $(\mathrm{P}<0.05)$ (Table III).

\section{Discussion}

The continuous development of preventive medicine and people's health care awareness have led to a significant reduction in the incidence of child typhoid, although the outbreak of typhoid fever in the rural and remote areas of China remains a problem (6). Typhoid has been defined as an acute gastrointestinal infectious disease. Pathogenic bacteria causing typhoid includes Salmonella typhimurium and Salmonella paratyphi A. In the last century, as ampicillin, chloramphenicol, and cotrimoxazole appeared and were used against typhoid fever, this disease was gradually controlled (7). Nonetheless by the end of the 20th century, multi-resistant Salmonella typhimurium strains began to emerge and made the treatment more complicated. The third generation cephalosporin and quinolone antibiotics were capable of improving the clinical symptoms, reducing the complications, and improving the long-term prognosis. However, the emergence of resistant strains created an obstacle and typhoid fever combined with toxic hepatitis, encephalitis, myocarditis, acute renal failure and other serious complications limited the application of antibiotics to some extent $(8,9)$.

Typhoid bacilli with their endotoxin can directly damage the cell membrane, thus harming myocardial cells. Following a Salmonella typhimurium infection, the patient's body enters a 
Table I. Comparison of the clinical efficiency in the two groups, $\mathrm{n}(\%)$.

\begin{tabular}{lcccc}
\hline Groups & Recovery, $\mathrm{n}(\%)$ & Effective, $\mathrm{n}(\%)$ & Ineffective, $\mathrm{n}(\%)$ & Total efficiency, $\mathrm{n}(\%)$ \\
\hline Observation group $(\mathrm{n}=27)$ & $13(48.1)$ & $12(44.4)$ & $2(7.4)$ & $25(92.5)$ \\
Control group $(\mathrm{n}=27)$ & $10(37.0)$ & $9(33.3)$ & $8(29.6)$ & $19(70.3)$ \\
$\chi^{2}$ & 2.358 & 2.413 & 2.856 & 3.412 \\
P-value & 0.032 & 0.034 & 0.028 & 0.017 \\
\hline
\end{tabular}

Table II. Comparison of the improvement rate of the syndrome in the two groups, $\mathrm{n}(\%)$.

\begin{tabular}{lcccc}
\hline Groups & Recovery, $\mathrm{n}(\%)$ & Effective, $\mathrm{n}(\%)$ & Ineffective, $\mathrm{n}(\%)$ & Total efficiency, $\mathrm{n}(\%)$ \\
\hline Observation group $(\mathrm{n}=27)$ & $14(51.9)$ & $10(37.0)$ & $3(11.1)$ & $24(88.9)$ \\
Control group $(\mathrm{n}=27)$ & $8(29.6)$ & $9(33.3)$ & $10(37.0)$ & $19(62.9)$ \\
$\chi^{2}$ & 3.526 & 0.856 & 2.845 & 3.956 \\
P-value & 0.024 & 0.632 & 0.031 & $<0.001$ \\
\hline
\end{tabular}

TCM, traditional Chinese medicine.

Table III. Comparison of changes observed in CK-MB and CRP levels in the two groups.

\begin{tabular}{lcccc}
\hline \multirow{2}{*}{ Groups } & \multicolumn{2}{c}{ CK-MB (U/l) } & \multicolumn{2}{c}{ CRP (mg/l) } \\
\cline { 2 - 4 } & Pre-treatment & Post-treatment & & Pre-treatment \\
\hline Observation group & $89.3 \pm 12.4$ & $23.5 \pm 6.8$ & $12.4 \pm 3.6$ & $5.8 \pm 2.1$ \\
Control group & $86.5 \pm 14.6$ & $45.2 \pm 5.1$ & $11.2 \pm 4.3$ & $7.7 \pm 1.9$ \\
t-test & 0.365 & 3.625 & 0.638 & 3.417 \\
P-value & 0.204 & 0.024 & 0.412 & 0.021 \\
\hline
\end{tabular}

CRP, C-reactive protein; CK-MB, creatinine kinase-MB.

hypersensitive state. During the course of the treatment a large number of lysed bacteria can release a considerable amount of endotoxin and allergenic debris into the bloodstream. Allergens have the capacity to cause severe allergic reactions in patients who are in hypersensitive state. This condition may lead to further damage to the cardiac muscle cells $(10,11)$.

Western medication for typhoid complicated by myocarditis was only limited to antibiotic therapy and symptomatic supportive therapy. On the other hand, Chinese medicine for typhoid achieved good symptomatic results on the symptoms of heart-yang deficiency (12). TCM held that typhoid was a 'damp-warm disease', which was characterized for damp-heat, slow onset, slow transformation, heat lingering, and long duration. In clinic, it was often shown in lienogastric syndromes (13). The disease was first recorded in 'the Internal Canon of Medicine', which described it as 'damp abundance leads to soggy diarrhea' (14). TCM for the treatment of typhoid was derived from damp-warm disease, with a focus on $q i$ leel, while paying attention to four NOs: i) no diaphoresis; ii) no salivation promotion; iii) no liquid increment; and iv) no bitter cold exposure. Only if the activities of $q i$ was regulated well, the body fluid may be regenerated, the thirst be relieved, dampness-heat be cleared, spleen-stomach be smoothened, and all symptoms disappeared (15).

'Ling Gui Long Mu soup' used in the present study has the capacity to inhibit the autoimmune problems, effectively inhibit the abnormal reactions in the body and achieving curative effects. The prescription of 'Ling Gui Long Mu soup' included Radix Paeoniae Alba which nourished the blood and eased the pains; cassia twig which relieved exterior syndromes, dispeled the cold, regulated the pulse, warmed the meridian, removed obstructions and activated yang; angelica was able to enrich and invigorate the blood, ease the pains and regulate menstruation; astragalus membranaceus that was sweet warm could invigorate vital energy and blood; American ginseng that is sweet cold could invigorate the lungs and decrease the internal heat, promote salivation and dispel tiredness; Cornu Antelopis that could diminish swelling and remove stasis, replenish blood and detoxify, sooth liver and subside interior winds; keel that could calm the breath and compose the spirit; ostracean that was salty in taste and cold-natured and could suppress liver-yang and calm the liver; liquorice that was sweet in taste and could tonify the spirits $(13,15,16)$. In the present study, we treated one group of the patients with 
'Ling Gui Long Mu soup' combined with Western medicine antibiotic therapy and the other group with pure Western medicine antibiotic therapy. The results showed that the total efficiency of the treatment for the patients treated with 'Ling Gui Long Mu soup' and antibiotics was significantly higher. The improvement rate of TCM group was significantly better, and the levels of the CRP and CK-MB after treatment was reduced more significantly when compared to the control group treated solely with Western medicine therapy. CK-MB is a sensitive enzymatic indicator released in the early period after a myocardial injury event. CRP level can reflect the inflammatory reactions in the body.

In conclusion, TCM 'Ling Gui Long Mu soup' can reduce the intensity of myocardial injury, significantly improve the clinical efficacy of child typhoid complicated by myocarditis, and improve the syndrome. Therefore, 'Ling Gui Long Mu soup' proved to be beneficial in clinical practice.

\section{References}

1. Buckle GC, Walker CLF and Black RE: Typhoid fever and paratyphoid fever: Systematic review to estimate global morbidity and mortality for 2010. J Glob Health 2: 10401, 2012.

2. Gilman RH, Terminel M, Levine MM, Hernandez-Mendoza P and Hornick RB: Relative efficacy of blood, urine, rectal swab, bone-marrow, and rose-spot cultures for recovery of Salmonella typhi in typhoid fever. Lancet 1: 1211-1213, 1975.

3. Allymehr M: Seroprevalence of Ornithobacterium rhinotracheale infection in broiler and broiler breeder chickens in West Azerbaijan Province, Iran. J Vet Med A Physiol Pathol Clin Med 53: 40-42, 2006.

4. Liu ZL, Liu ZJ, Liu JP, Yang M and Kwong J: Herbal medicines for viral myocarditis. Cochrane Database Syst Rev 7: CD003711, 2010.

5. Banani M, Pourbakhsh SA and Deihim AH: Antibiotic sensitivity of Ornithobacterium rhinotracheale isolates associated with respiratory diseases. Arch Razi Ins 58: 111-117, 2004.

6. Yang HH, Wu CG, Xie GZ, Gu QW, Wang BR, Wang LY, Wang HF, Ding ZS, Yang Y, Tan WS, et al: Efficacy trial of Vi polysaccharide vaccine against typhoid fever in south-western China. Bull World Health Organ 79: 625-631, 2001.
7. García M: Typhoid fever in nineteenth-century Colombia between medical geography and bacteriology. Med Hist 58: 27-45, 2014.

8. Kaljee LM, Pach A, Thriemer K, Ley B, Ali SM, Jiddawi M Puri M, von Seidlein L, Deen J, Ochiai L, et al: Utilization and accessibility of healthcare on Pemba Island, Tanzania: implications for health outcomes and disease surveillance for typhoid fever. Am J Trop Med Hyg 88: 144-152, 2013.

9. Mweu E and English M: Typhoid fever in children in Africa. Trop Med Int Health 13: 532-540, 2008.

10. Moore CE, Pan-Ngum W, Wijedoru LP, Sona S, Nga TV, Duy PT, Vinh PV, Chheng K, Kumar V, Emary K, et al: Evaluation of the diagnostic accuracy of a typhoid $\operatorname{IgM}$ flow assay for the diagnosis of typhoid fever in Cambodian children using a Bayesian latent class model assuming an imperfect gold standard. Am J Trop Med Hyg 90: 114-120, 2014.

11. Andualem G, Abebe T, Kebede N, Gebre-Selassie S, Mihret A and Alemayehu H: A comparative study of Widal test with blood culture in the diagnosis of typhoid fever in febrile patients. BMC Res Notes 7: 653, 2014.

12. Khoo SK, Petillo D, Parida M, Tan AC, Resau JH and Obaro SK: Host response transcriptional profiling reveals extracellular components and ABC (ATP-binding cassette) transporters gene enrichment in typhoid fever-infected Nigerian children. BMC Infect Dis 11: 241, 2011.

13. Ley B, Mtove G, Thriemer K, Amos B, von Seidlein L, Hendriksen I, Mwambuli A, Shoo A, Malahiyo R, Ame SM, et al: Evaluation of the Widal tube agglutination test for the diagnosis of typhoid fever among children admitted to a rural hdospital in Tanzania and a comparison with previous studies. BMC Infect Dis 10: 180, 2010.

14. Xie DY and Chen RX: The two-step location method of acupoint in Internal Canon of Medicine and its clinical application. Zhongguo Zhen Jiu 34: 979-982, 2014 (In Chinese).

15. Wijedoru LPM, Kumar V, Chanpheaktra N, Chheng K, Smits HL, Pastoor R, Nga TV, Baker S, Wuthiekanun V, Peacock SJ, et al: Typhoid fever among hospitalized febrile children in Siem Reap, Cambodia. J Trop Pediatr 58: 68-70, 2012.

16. Khanam F, Sayeed MA, Choudhury FK, Sheikh A, Ahmed D, Goswami D, Hossain ML, Brooks A, Calderwood SB, Charles RC, et al: Typhoid fever in young children in Bangladesh: clinical findings, antibiotic susceptibility pattern and immune responses. PLoS Negl Trop Dis 9: e0003619, 2015. 